\title{
LEPTOCYLINDRUS MINIMUS GRAN (BACILLARIOPHYCEAE): MORFO- LOGIA Y DISTRIBUCION EN CHILE
}

\author{
LEPTOCYLINDRUS MINIMUS GRAN (BACILLARIOPHYCEAE): \\ MORPHOLOGY AND DISTRIBUTION IN CHILE
}

\author{
Patricio Rivera $^{1}$, Fabiola Cruces $^{1} \&$ Alejandro Clement ${ }^{2}$
}

RESUMEN

Sobre la base de material recolectado en Calbuco, Chiloé, se confirma la presencia en Chile de Leptocylindrus minimus Gran (Bacillariophyceae).

Palabras Claves: Leptocylindrus minimus Gran, Bacillariophyceae, Chile.

\section{INTRODUCCION}

De las especies que comprende el género Leptocylindrus Cleve (sin considerar a Dactyliosolen mediterraneus (Peragallo) Peragallo, cuya inclusión por algunos investigadores en el género Leptocylindrus es cuestionable), L. danicus Cleve es la única que ha sido reiteradamente recolectada y citada por diferentes autores a lo largo de toda la costa chilena (Rivera 1983, Rivera et al. 1990). A partir de 1981, otra especie del género, L. minimus Gran, empieza a ser señalada para las aguas australes del país (Lembeye 1981), y en 1989, 1993 y 1994 se la encuentra en altas concentraciones en la zona central y sur del Archipiélago de Chiloé, y se asocia su presencia con la muerte de salmones en cautiverio (Clement 1994).

${ }^{1}$ Departamento de Botánica, Universidad de Concepción, Casilla 160-C, Concepción. E-mail: privera@udec.cl

${ }^{2}$ Plancton Andino, Casilla 823, Puerto Montt.

\section{ABSTRACT}

Based on material collected in Calbuco, Chiloé, the presence of Leptocylindrus minimus Gran (Bacillariophyceae) in Chile is confirmed.

Keywords: Leptocylindrus minimus Gran, Bacillariophyceae, Chile.

Sin embargo, Hargraves (1990) luego de estudiar la morfología y distribución mundial de $L$. minimus, señala que aunque puede ser considerada como una especie cosmopolita, estaría ausente de las aguas del Pacífico Suroriental incluyendo las subantárticas y antárticas. Como las citas de $L$. minimus para Chile fueron realizadas sobre la base de observaciones hechas exclusivamente con microscopía fotónica, no existiendo dibujos, fotografías o descripciones de los individuos encontrados, nos pareció de gran interés verificar la identidad de la especie involucrada, utilizando para ello técnicas de microscopía electrónica.

El material estudiado fue recolectado en marzo de 2000 en Calbuco-Chiloé, y se encuentra depositado en la Colección de Diatomeas de la Universidad de Concepción (DIAT-CONC M-3192). Con fines comparativos, se analizó también material correspondiente a Leptocylindrus danicus, recolectado en la costa chilena (Bahía de Concepción y Bahía de Coliumo) y depositado en esta misma Colección (DIAT-CONC M 2263, 2524). 
El presente trabajo confirma la presencia de L. minimus en Chile. Algunas características morfológicas de esta especie permiten diferenciarla fácilmente de L.danicus, incluso usando técnicas de microscopía fotónica.

\section{DESCRIPCION}

Leptocylindrus minimus Gran, p. 72, Fig. 5 (1915) Figs. 1-7

Referencias: Hustedt 1930, p. 560, Fig. 321; Lebour 1930, p. 78, Fig. 52c; Hasle 1975, p. 125, Fig. 142; Hargraves 1990, pp. 47-60; Hasle \& Syvertsen 1996, pp. 93, 95, Lám. 14.

Células cilíndricas, muy poco silicificadas, usualmente solitarias aunque también se las encontró unidas por la cara valvar en cadenas rectas, cortas, de hasta tres células (Fig. 1). Diámetro: 2-5.2 $\mu \mathrm{m}$; eje pervalvar: 22-48 $\mu \mathrm{m}$. Uno a dos (generalmente dos) cloroplastos de contorno alargado en cada célula. Cingulum formado por numerosas bandas del tipo media-banda, de contorno trapezoidal, 1-3 $\mu \mathrm{m}$ de ancho, todas con la misma estructura consistente en líneas verticales, algo curvadas, de pequeñas aréolas, 11-14 en $1 \mu \mathrm{m}$ (Figs. 2-6). Cada individuo posee valvas convexas (Figs. 3-4), pero una de ellas es cóncava en su parte media (Figs. 2, 5), lo que facilita la unión entre dos células contiguas; el centro de cada valva parece estar más silicificado (Fig. 7). En la unión de la cara valvar con el manto se encuentra un anillo de pequeñas elevaciones silícicas, más anchas que altas, 2-3 en $1 \mu \mathrm{m}$ (Figs. 2-5, 7); un segundo anillo de elevaciones aún más reducidas, 5-6 en $1 \mu \mathrm{m}$, está presente sólo en algunas valvas a corta distancia del margen valvar (Fig. 5). La cara valvar y el manto valvar están ornamentados con líneas radiales de pequeñas aréolas, 8-13 en 1 $\mu \mathrm{m}$ (Figs. 3-4, 7). Proceso labiado ausente.

\section{OBSERVACIONES}

Existen ligeras diferencias entre las características morfológicas observadas en el material chileno y el material analizado por Hargraves (1990). Este autor describe que las cadenas son derechas o levemente onduladas; estas últimas no fueron encontradas en el material estudiado, debido posiblemente, a que las cadenas estaban estructuradas por un número no superior a tres células. Tampoco fue observado en nuestro material un "cluster" de aréolas en la parte central de la valva, rodeado de un anillo silícico nítido, característica igualmente no constante en el material de Hargraves. Otras desigualdades se relacionan con un diámetro valvar ligeramente mayor, un menor número de aréolas y de espinas sobre la cara valvar, y con la existencia de un patrón definido de distribución de las aréolas en las bandas del cingulum (Tabla I). No obstante, se debe tener presente que, hasta la fecha, la descripción de Hargraves para esta especie, basada en material recolectado en la Bahía Narragansett, Rhode Island (aprox. 41 $25^{\circ} \mathrm{N}$ $71^{\circ} 15^{\prime} \mathrm{W}$ ), era la única que incluía observaciones con microscopía electrónica. Por lo tanto, el presente estudio complementa el conocimiento de las características morfológicas de esta especie y revela el rango de variación a que están sujetas algunas de ellas.

Ninguna de la valvas de L. minimus observadas en el presente estudio exhibió un poro subcentral, el cual siempre está presente en la otra especie chilena, L. danicus Cleve (Figs. 8-11). Además, este último taxon difiere de L. minimus, por sus frústulos más silicificados, por su mayor diámetro valvar y por la presencia de numerosos cloroplastos de reducido tamaño en cada célula, caracteres reconocibles con microscopía fotónica. $\mathrm{Se}$ diferencia también por sus aréolas de mayor tamaño (Tabla I) y por la presencia de un grupo de aréolas en el área central, separadas de las restantes por un anillo silícico (Figs. 10-11). Aunque no fueron encontradas esporas de resistencia en el material estudiado, su forma es diferente en ambas especies (Hargraves 1990).

Leptocylindrus minimus parece estar distribuído en las aguas de Chile entre los $39^{\circ} \mathrm{S}$ y $\operatorname{los} 52^{\circ} \mathrm{S}$. Si bien la única referencia segura de su presencia corresponde al material estudiado en el presente trabajo (Canal Caicahén, Calbuco, Chiloé, $\left.41^{\circ} 47^{\prime} \mathrm{S}-73^{\circ} 10^{\prime} \mathrm{W}\right)$, el taxón fue señalado por primera vez por Lembeye (1981) para el Seno Unión, Magallanes (apróx. 5205'S-74³0'W), por Lembeye y Campodónico (1984) para el Seno de Reloncaví (aprox. 4140'S-7250W), por Toro (1984) para el Estuario del Río Queule (apróx. $\left.39^{\circ} 15^{\prime} \mathrm{S}-73^{\circ} 09^{\prime} \mathrm{W}\right)$, y por Clement y Lembeye (1993) y Clement (1994) para distintas localidades a lo largo de la Isla de Chiloé (entre los $42^{\circ} \mathrm{S}$ y $43^{\circ} \mathrm{S}$ ). 

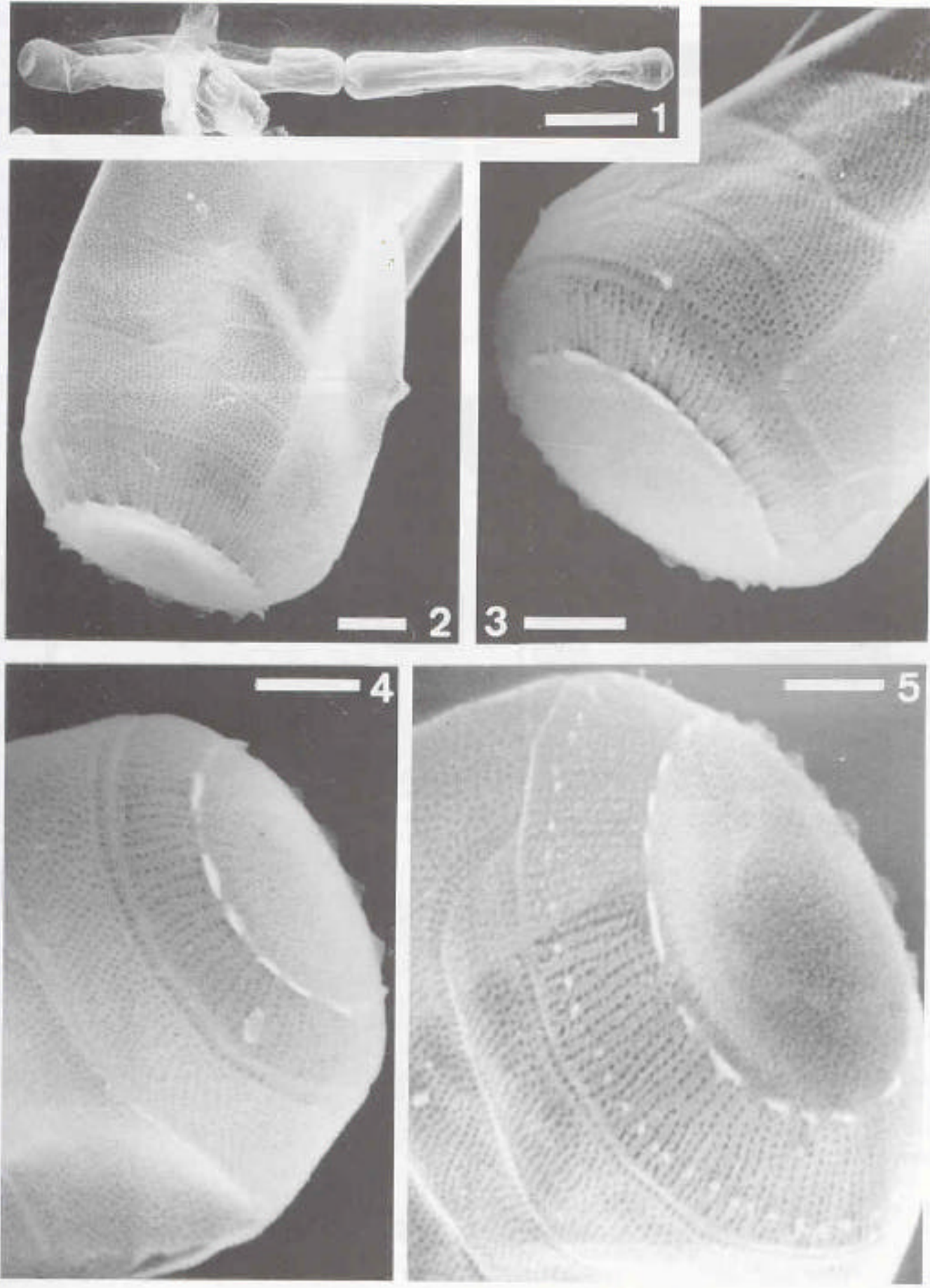

Figs. 1-5. Leptocylindrus minimus, SEM. Escala $1 \mu \mathrm{m}$, excepto Fig. $1=10 \mu \mathrm{m}$. Fig. 1. Cadena de dos células. Fig. 2. Cara valvar cóncava con elevaciones silícicas en la unión con el manto valvar. Cíngulum formado por numerosas medias-bandas. FIg. 3. Cara valvar convexa. Detalle de bandas del cingulum. Fig. 4. Cara valvar, líneas radiales de aréolas sobre la valva. Fig. 5. Valva cóncava, con un segundo anillo de elevaciones silícicas cerca del margen valvar. 
Gayana Bot. 59(1), 2002
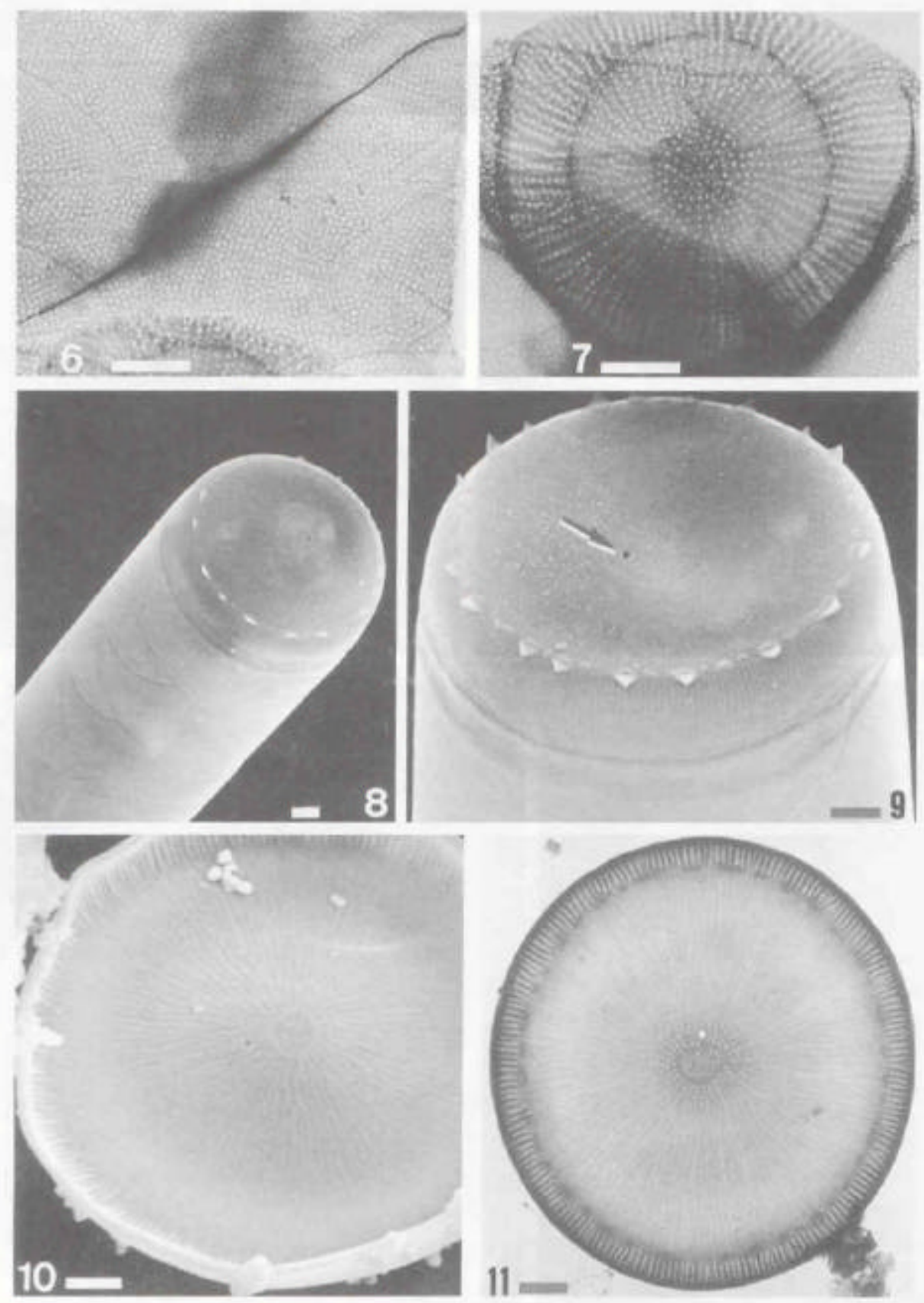

FIGs. 6-7. Leptocylindrus minimus, TEM. Escala $1 \mu \mathrm{m}$. FIG. 6. Detalle de bandas del cingulum. FIg. 7. Valva con líneas radiales de aréolas. Figs. 8-11. Leptocylindrus danicus. Escala $1 \mu \mathrm{m}$. Figs. 8-10, SEM; FIG. 11, TEM. Fig. 8. Vista de la cara valvar y del cingulum. FIG. 9. Poro ubicado en la parte subcentral de la valva (flecha). Fig. 10. Vista valvar interna. Líneas radiales de aréolas y poro subcentral. FIG. 11. Cluster central de aréolas rodeado por anillo silícico; poro subcentral. 
Leptocylindrus minimus (Bacillariophyceae) en Chile: RIVERA, P. ET AL.

TABLA I. Información morfométrica de las especies chilenas de Leptocylindrus.

\begin{tabular}{|c|c|c|c|}
\hline & $\begin{array}{c}\text { L. minimus } \\
\text { (presente estudio) }\end{array}$ & $\begin{array}{c}\text { L. minimus } \\
\text { (Hargraves 1990) }\end{array}$ & $\begin{array}{c}\text { L. danicus } \\
\text { (presente estudio) }\end{array}$ \\
\hline Diámetro $(\mu \mathrm{m})$ & $2-5,2$ & $1,5-4,5$ & $6-14$ \\
\hline $\begin{array}{l}\text { Número de cloroplastos por } \\
\text { célula }\end{array}$ & $1-2$ & $1-2$ & numerosos \\
\hline $\begin{array}{l}N^{0} \text { de líneas de aréolas en } \\
\text { valva }(\text { en } 1 \mu \mathrm{m})\end{array}$ & $8-13$ & $13-18$ & $5-9$ \\
\hline $\begin{array}{l}\text { No de elevaciones silícicas } \\
\text { entre cara valvar y manto (en } \\
1 \mu \mathrm{m} \text { ) }\end{array}$ & $2-3$ & $3-5$ & $1-3$ \\
\hline $\begin{array}{l}\text { Poro ubicado en la parte } \\
\text { subcentral de la valva }\end{array}$ & No & No & Sí \\
\hline $\begin{array}{l}\text { Cluster de aréolas al centro de } \\
\text { la valva }\end{array}$ & No & Si / No & Sí \\
\hline
\end{tabular}

\section{AGRADECIMIENTOS}

Agradecemos la gran ayuda prestada por el personal del Laboratorio de Microscopía Electrónica de la Universidad de Concepción, especialmente de los Srs. Hugo Pacheco, Raúl Alarcón y Julio Pugin.

\section{BIBLIOGRAFIA}

Clement, A. 1994. Harmful blooms of Leptocylindrus minimus in Southern Chile. Harmful Algae News, 8: 1 .

Clement, A. \& G. Lembeye. 1993. Phytoplankton Monitoring Program in the Fish Farming Region of South Chile. In: Smayda, T. \& Y. Shimizu (Eds.), Toxic Phytoplankton Blooms in the Sea, pp. 223-228, Elsevier Science Publishers B.V.

Gran, H.H. 1915. The plankton production in the north European waters in the spring of 1912. Cons. Perm. Int. L'explor. Mer, Bull. Plankton pour Lanée, pp. 1-142.

Hargraves, P.E. 1990. Studies on marine plankton diatoms. V. Morphology and distribution on Leptocylindrus minimus Gran. Beih. Nova Hedwigia 100: 47-60.

HaSLE, G.R. 1975. Some Living Marine Species of the Diatom Family Rhizosoleniaceae. Beih. Nova Hedwigia 53: 99-152.
Hasle, G.R. \& E. Syvertsen. 1996. Marine Diatoms. In: Tomas, C. (Ed.), Identifying Marine Diatoms and Dinoflagellates, pp. 5-385, Academic Press, Inc.

Hustedt, F. 1930. Die Kieselalgen Deutschlands, Österreichs und der Schweiz. L. Rabenhorst Kryptogamen-Flora, 7(1): 1-920.

Lebour, M.V. 1930. The Plankton Diatoms of Northern Seas. Ray Society, London, 244 pp.

Lembeye, G. 1981. Estructura del Fitoplancton asociado a la presencia del veneno paralítico de los mariscos en Seno Unión y áreas adyacentes (Magallanes, Chile), 1981. Ans. Inst. Pat., Punta Arenas (Chile), 12: 277-288.

Lembeye, G. \& I. Campodonico. 1984. First Record Bloom of the Dinoflagellate Prorocentrum micans Ehr. in South-Central Chile. Botanica Marina 27: 491-493.

Rivera, P. 1983. A Guide for References and Distribution for the Class Bacillariophyceae in Chile Between $18^{\circ} 28^{\prime} \mathrm{S}$ and $58^{\circ} \mathrm{S}$. Bibliotheca Diatomologica, Band 3, 386 pp.

Rivera, P., M. Gebauer \& H.L. Barrales. 1990. A Guide for References and Distribution for the Class Bacillariophyceae in Chile between $18^{\circ} 28^{\prime} \mathrm{S}$ and $58^{\circ}$ S. Part II. Data from 1982 to 1988 . Gayana Bot. 46(3-4): 155-198.

Toro, J. 1984. Determinación de las fluctuaciones mensuales de la abundancia y de la biomasa fitoplanctónica en el Estuario del Río Queule (Chile, IX Región). Rev. Biol. Mar., Valparaíso, 20: 23-37. 